Check for updates

Cite this: RSC Adv., 2018, 8, 11403

\title{
Oxygen insensitive thiol-ene photo-click chemistry for direct imprint lithography of oxides $\uparrow$
}

\author{
Ravikiran Nagarjuna, ${ }^{a}$ Mohammad S. M. Saifullah ${ }^{\mathrm{b}}$ and Ramakrishnan Ganesan (D)*a
}

UV-nanoimprint lithography (UV-NIL) is a promising technique for direct fabrication of functional oxide nanostructures. Since it is mostly carried out in aerobic conditions, the free radical polymerization during imprinting is retarded due to the radical scavenging ability of oxygen. Therefore, it is highly desirable to have an oxygen-insensitive photo-curable resin that not only alleviates the requirement of inert conditions but also enables patterning without making substantial changes in the process. Here we demonstrate the formulation of metal-containing resins that employ oxygen-insensitive thiol-ene photo-click chemistry. Allyl acetoacetate (AAAC) has been used as a bifunctional monomer that, on one hand, chelates with the metal ion, and on the other hand, offers a reactive alkene group for polymerization. Pentaerythritol tetrakis(3-mercaptopropionate) (PETMP), a four-arm thiol derivative, is used as a crosslinker as well as an active component in the thiol-ene photo-click chemistry. The FT-IR analyses on the metal-free and metal-containing resin formulations revealed that the optimum ratio of alkene to thiol is $1: 0.5$ for an efficient photo-click chemistry. The thiol-ene photo-click chemistry has been successfully demonstrated for direct imprinting of oxides by employing $\mathrm{TiO}_{2}$ and $\mathrm{Ta}_{2} \mathrm{O}_{5}$ as candidate systems. The imprinted films of metal-containing resins were subjected to calcination to obtain the corresponding patterned metal oxides. This technique can potentially be expanded to other oxide systems as well.

Received 26th February 2018 Accepted 16th March 2018

DOI: $10.1039 / \mathrm{c} 8 \mathrm{ra01688g}$

rsc.li/rsc-advances photo-addition. ${ }^{13-16}$ Hydrothiolation via photo-clicking is attractive as it proceeds well at room temperature reaction conditions. In a typical thiol-ene click reaction, the photogenerated electron from a photo-initiator is transferred to the thiol group to produce a thiyl radical, which adds across a carbon-carbon double bond and thus transferring the radical to the alkene. Abstraction of a hydrogen radical from the neighborhood thiol to this carbon radical accomplishes the hydrothiolation, while simultaneously propagating the chain reaction..$^{9,17}$

Fabrication of micro/nanostructures of oxides is of paramount importance in various applications including photoelectrochemical cells, ${ }^{18}$ photovoltaic cells, ${ }^{19}$ sensors,${ }^{20}$ and catalysis. ${ }^{21}$ Several techniques including photolithography, ${ }^{22,23}$ electron-beam lithography, ${ }^{24,25}$ electro-hydrodynamic lithography, ${ }^{26,27}$ direct write assembly, ${ }^{28}$ selective surface wetting, ${ }^{29}$ dip-pen nanolithography, ${ }^{30,31}$ nanoimprint lithography (NIL) ${ }^{32-34}$ among others ${ }^{35,36}$ have been utilized towards fabrication of metal oxide micro/nanostructures. Each of these techniques has its own merits as well as limitations. Among the various available techniques, NIL has been regarded as the next generation fabrication approach due to its advantages such as simplicity, low-cost, high-fidelity, high-resolution, nondependence on the optical diffraction limit, compatibility with various substrates and ability to imprint arbitrary structures over flat and curved surfaces. ${ }^{37-40}$ The descendants of NIL like
${ }^{a}$ Department of Chemistry, Birla Institute of Technology and Science (BITS) Pilani, Hyderabad Campus, Jawahar Nagar, Shameerpet Mandal, Hyderabad, 500078, India. E-mail: ram.ganesan@hyderabad.bits-pilani.ac.in

${ }^{b}$ Institute of Materials Research and Engineering, A*STAR (Agency for Science, Technology, and Research), 2 Fusionopolis Way, \#08-03 Innovis, Singapore 138634, Republic of Singapore

$\dagger$ Electronic supplementary information (ESI) available. See DOI: 10.1039/c8ra01688g 
step-and-flash, ${ }^{41-43}$ roll-to-roll ${ }^{44}$ and roll-to-flat ${ }^{45}$ imprint lithography techniques have augmented its high-throughput capability while maintaining high-precision. The NIL process can be broadly classified into two categories: thermal and UV. Thermal NIL requires temperature cycling, which reduces the throughput, affects the mold life, and leads to line-width variation owing to the differences in thermal expansion coefficients of the mold, substrate, and resist. ${ }^{46}$ On the other hand, UV-NIL is an attractive room temperature process that does not require any temperature cycling, and thereby allows high-throughput patterning.

As opposed to the fabrication of polymeric micro/ nanostructures, fabrication of oxides has inherent challenges in the materials design as the metal-containing polymers and ceramics do not possess workable characteristics for direct patterning. Therefore, the material design has to be customized as per the requirement of each technique. In the case of nanoimprinting, sol-gel approach was initially employed that utilized hydrolysis followed by condensation of metal alkoxides or halides. ${ }^{33,47-49}$ The conventional sol-gel approach suffered low yield due to the lack of mechanical strength of the imprints during the demolding step. Nanoparticle suspensions have also been used for direct nanoimprinting with a soft PDMS mold. ${ }^{50}$ This approach has been used to directly fabricate threedimensional structures of multicomponent oxides like indium tin oxide and nickel ferrite. ${ }^{51,52}$ Photosensitive ethylhexanoatebased oxide patterning has been reported that utilizes photobreakable precursors rather than photo-curable monomers. ${ }^{34}$ Recently, polymerizable sol-gel approach (PSG) has emerged as a promising method for rapid direct nanoimprinting of a host of metal oxides such as $\mathrm{TiO}_{2}, \mathrm{ZrO}_{2}, \mathrm{Hf}_{2} \mathrm{O}_{5}$ amongst others. ${ }^{46,53,54}$ The PSG approach utilizes metal-methacrylate liquid precursors that undergo in situ thermal- or photo-induced free radical polymerization during the nanoimprinting step. One of the major advantages of this approach is the spin-coatable liquid phase metal-containing resin that can easily fill up the pattern features of the mold at near-ambient pressure due to capillary action. Polymerization solidifies the metal-containing precursor and decreases the surface energy to facilitate easy and clean demolding. This approach has been found to harness the advantages and obviates the limitations of the conventional sol- gel approach. The PSG approach has been shown to be suitable for high-throughput step-and-flash imprint lithography for various oxides ${ }^{55}$ and to synthesize supported oxide photo-catalysts. ${ }^{56,57}$ Despite the high potential, the PSG approach based on free radical polymerization of methacrylates is vulnerable to radical scavenging by oxygen that could affect the degree of polymerization, which in turn could effect on the quality of imprinting. This warrants purging/bubbling of inert gas into the metal-containing resin before patterning in order to eliminate any dissolved oxygen.

As thiol-ene click chemistry can fulfil the requirements such as oxygen-insensitivity, spin-coatability, and room temperature photo-crosslinking, it is deemed to be attractive for UV-NIL. ${ }^{58-60}$ This chemistry has recently been shown for its potential for imprinting polymeric structures over flat and curved surfaces. ${ }^{61}$ However, the merits of the thiol-ene photo-click chemistry have so far not been realized for oxide nanoimprinting. Here, we show the augmentation of the PSG approach by employing the oxygen-insensitive thiol-ene click chemistry towards fabrication of metal oxide micro/nanostructures (Scheme 1). The design of the photo-curable metal-containing resin, its photocurability and patternability are discussed by choosing titanium alkoxide as the precursor to fabricate $\mathrm{TiO}_{2}$ nanostructures. $\mathrm{TiO}_{2}$ was chosen as the principal oxide candidate, as it is one of the most studied semiconducting oxides that possesses huge potential for various applications including energy, health, and environment. ${ }^{62-64}$ Finally, the thiol-ene approach was also studied for patterning $\mathrm{Ta}_{2} \mathrm{O}_{5}$ in order to ascertain its potential towards structuring other metal oxides.

\section{Experimental section}

\subsection{Materials and methods}

Titanium(Iv) isopropoxide, tantalum(v) ethoxide, allyl acetoacetate (AAAc), pentaerythritol tetrakis(3-mercaptopropionate) (PETMP), 2-(methacryloyloxy)ethyl acetoacetate (MAEAA), 2hydroxy-2-methylpropiophenone (HMP), $1 \mathrm{H}, 1 \mathrm{H}, 2 \mathrm{H}, 2 \mathrm{H}$-perfluorodecyltrichlorosilane, and toluene were purchased from Sigma Aldrich and used as-received. Polydimethylsiloxane (PDMS) stamps were fabricated from Sylgard 184 purchased from Sigma Aldrich.

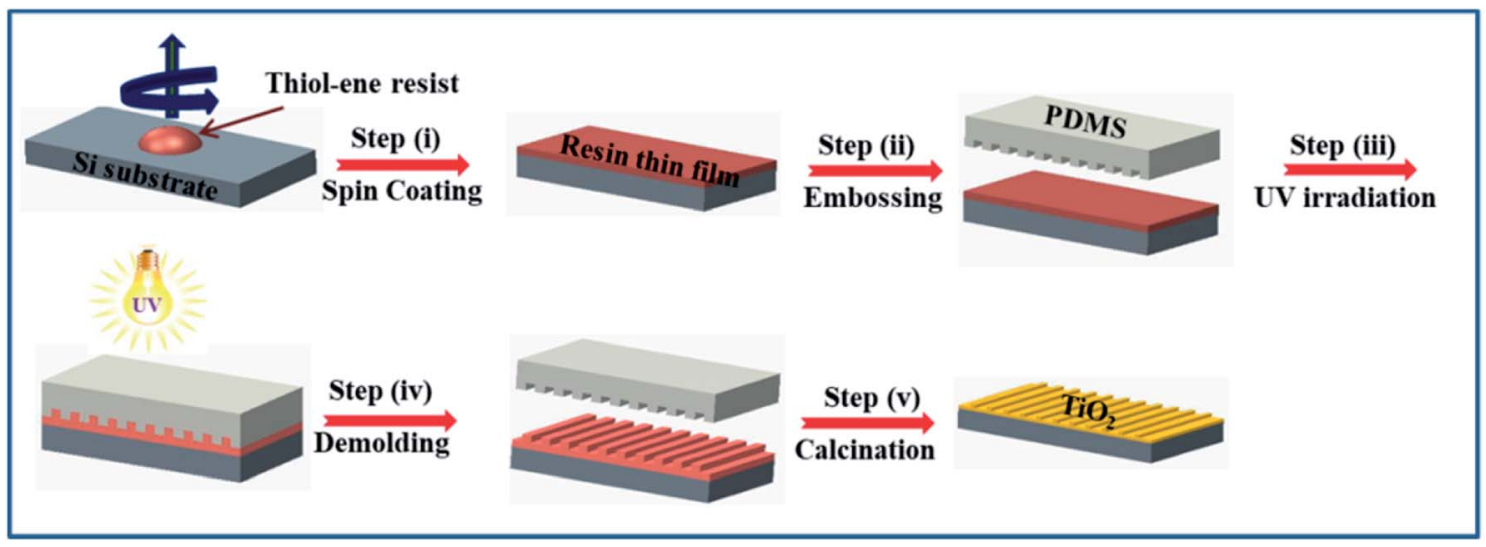

Scheme 1 Schematic representation of the overall process involving thiol-ene click chemistry for imprinting of metal oxides. 


\subsection{Resin formulation}

The $\mathrm{TiO}_{2}$ resists were prepared using requisite amounts of precursors as mentioned in Table 1 . Briefly, one equivalent of $\mathrm{Ti}(\mathrm{i}-\mathrm{PrO})_{4}$ was added and thoroughly stirred with 4 equivalents of AAAc in a glass vial inside a nitrogen glove box. The color of the solution rapidly turned into red that indicated the formation of Ti(AAAc) $)_{4}$ complex. To this complex, either half equivalent or one equivalent of the PETMP cross-linker was added and the resultant mixture was shaken well on a vortex stirrer for 2 min to obtain a clear solution. To this mixture, $3 \mathrm{wt} \%$ (with respect to the AAAc and PETMP) of HMP photo-initiator was added to formulate the imprintable resin, which was diluted with toluene in $1: 1$ (wt/wt) ratio to decrease the viscosity. The $\mathrm{Ta}_{2} \mathrm{O}_{5}$ resist was also prepared in a similar manner by taking tantalum(v) ethoxide as the alkoxide source.

\subsection{Imprinting of metal oxide}

First, PDMS stamps were fabricated following the standard procedure from Sylgard 184. Briefly, the silicon rubber base prepolymer and cross-linker of Sylgard 184 were thoroughly mixed in $10: 1$ weight ratio and the resulting mixture was poured over polycarbonate master mold kept in a Petri dish. This assembly was degassed in a vacuum desiccator to remove any trapped air bubbles. The assembly was the subjected to $70{ }^{\circ} \mathrm{C}$ for $1 \mathrm{~h}$ to induce cross-linking of the precursor. Finally, the PDMS elastomeric stamp was peeled off from the master mold. The fabricated PDMS stamps were treated with $1 H, 1 H, 2 H, 2 H$-perfluorodecyltrichlorosilane for $5 \mathrm{~h}$ inside an evacuated desiccator in order to decrease the surface energy that facilitates easy demolding. The water contact angle of the fluorinated PDMS stamp was found to be $\sim 130^{\circ}$ as opposed to $105^{\circ}$ for freshly prepared PDMS, indicating the reduction in surface energy.

In a typical nanoimprinting process, the metal-containing resin solution was spin-coated over a silicon wafer $(20 \mathrm{~mm} \times$ $20 \mathrm{~mm}$ ) at $1000 \mathrm{rpm}$ for $45 \mathrm{~s}$. The PDMS mold was then placed on top of the wet thin film and a slight pressure was applied. This assembly was subjected to UV irradiation with a $\mathrm{Hg}$ vapor lamp $(125 \mathrm{~W})$ for $20 \mathrm{~min}$ to induce crosslinking of the monomers. After UV exposure, the PDMS stamp was carefully demolded and the imprints were subjected to calcination at designated temperatures for $1 \mathrm{~h}$ to obtain metal oxide micro/ nanostructures.

\subsection{Characterization}

The thiol-ene photoreaction was following using FT-IR spectrophotometer (JASCO FTIR 4200). TGA analyses were carried

Table 1 Proportions of various components used in the different formulations

\begin{tabular}{lllll}
\hline $\begin{array}{l}\text { Sample } \\
\text { code }\end{array}$ & $\begin{array}{l}\text { TIPO } \\
(\mathrm{mmol})\end{array}$ & $\begin{array}{l}\text { AAAc } \\
(\mathrm{mmol})\end{array}$ & $\begin{array}{l}\text { PETMP } \\
(\mathrm{mmol})\end{array}$ & $\begin{array}{l}\text { HMP } \\
(\mathrm{mmol})\end{array}$ \\
\hline TA & 1.0 & 4.0 & 0.0 & 0.156 \\
TAP-0.5 & 1.0 & 4.0 & 0.50 & 0.208 \\
TAP-1 & 1.0 & 4.0 & 1.00 & 0.277
\end{tabular}

out using Shimadzu DTG-60 differential thermal analyzer in air atmosphere to follow the degradation profile and the residual oxide mass present in the metal-containing resins. XRD patterns of the thin film samples calcined at different temperatures were measured at a scan rate of $1^{\circ} \min ^{-1}$ using Rigaku Ultima IV X-ray diffractometer equipped with $\mathrm{Cu} \mathrm{K}_{\alpha}(\lambda=1.5418$ $\AA$ ). Diffuse reflectance spectroscopy analysis of the thin film samples calcined at different temperatures was performed using JASCO V-670 UV-visible spectrophotometer. The surface morphology of the imprints before and after calcination was characterized using a Carl-Zeiss ULTRA-55 field emission scanning electron microscope (FE-SEM).

\section{Results and discussion}

A judicious choice of precursors is the key to successful implementation of the thiol-ene click chemistry to realize oxide nanofabrication. In this regard, we chose pentaerythritol tetrakis(3-mercaptopropionate) (PETMP) as the cross-linker that has four thiol arms. Allyl acetoacetate (AAAc) was chosen as the bifunctional reagent that on one hand chelates with the metal center through the acetoacetate group and on the other hand possesses the reactive alkene group for the click reaction. When the metal center is chelated with more than one AAAc, it could also act as a cross-linker, which would lead to the formation of highly branched polymeric network. The chemical structures of the various components used in this study are shown in Fig. 1.

To probe the thiol-ene click reaction under UV irradiation, we formulated a resin containing PETMP and AAAc in $1: 4$ ratio mixed with $3 \mathrm{wt} \%$ of 2-hydroxy-2-methylpropiophenone (HMP) photo-initiator. This metal-free resin was spin-coated over a precleaned silicon wafer and studied with the Fourier transform infrared (FT-IR) spectroscopy to follow the thiol-ene photo-click chemistry (Fig. 2a). As seen from the figure, the characteristic -SH stretching peak was observed at $2570 \mathrm{~cm}^{-1}$, confirming the presence of free thiol groups in the resin formulation. In addition, an intense peak at $1738 \mathrm{~cm}^{-1}$ due to the carbonyl group stretching and a weak peak at $1632 \mathrm{~cm}^{-1}$ due to the alkene group were also observed. After subjecting the film to $20 \mathrm{~min}$ of UV irradiation, it remained wet, indicating poor

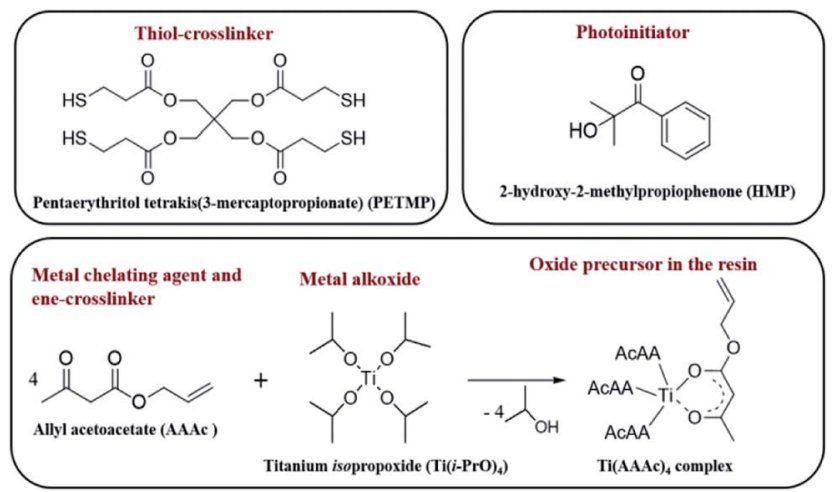

Fig. 1 Chemical structures of the various components used for imprinting of metal oxides. 

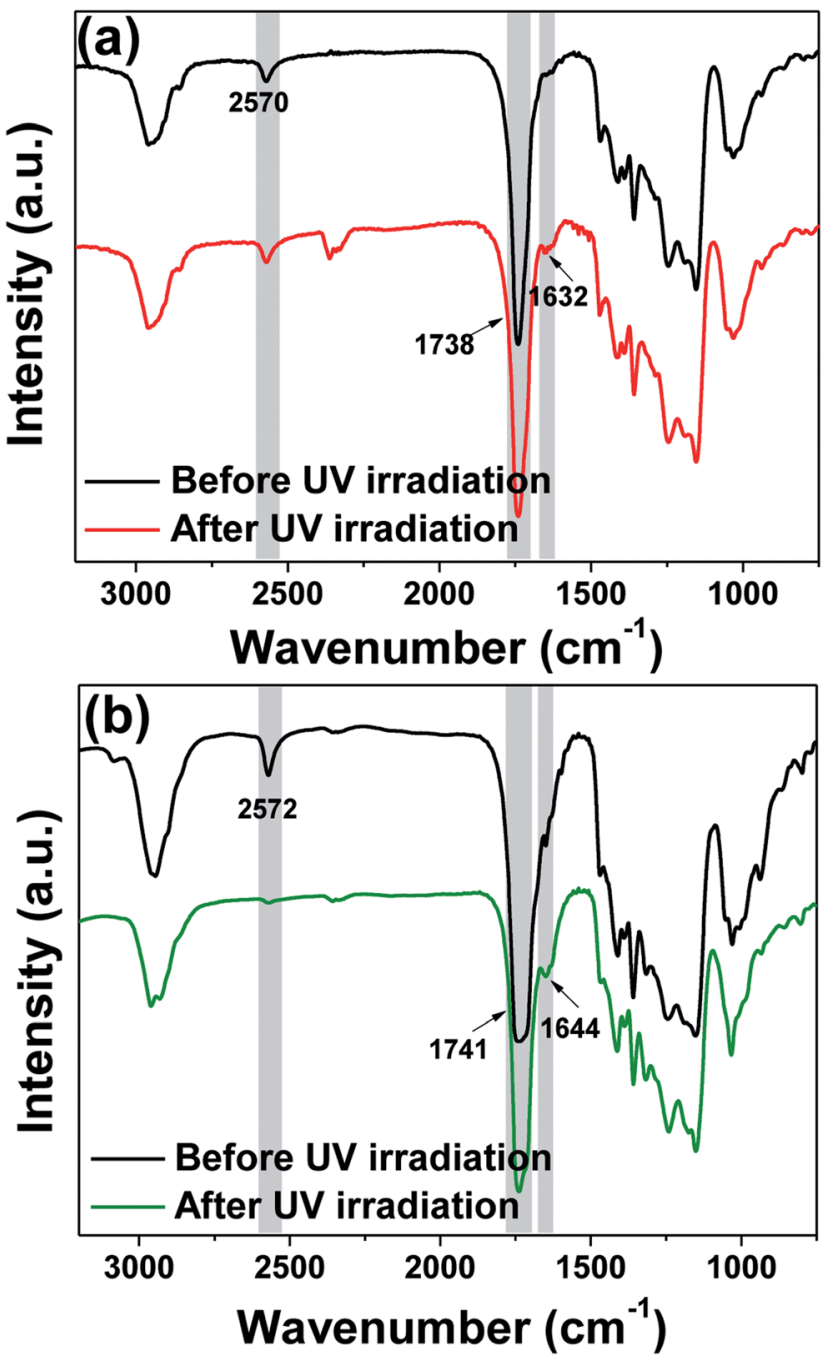

Fig. 2 FT-IR analyses of thiol-ene photo-curing of resins containing PETMP and AAAc in the ratio of (a) $1: 4$ (TAP-1) and (b) $1: 8$ (TAP-0.5) showing that the latter composition is amenable to the click chemistry.

efficiency of the thiol-ene click-reaction in this system. The FTIR spectrum obtained after irradiation was almost identical to that before UV irradiation. We postulated that this could be due to the unfavorably positioned functional groups or reduced probability of stoichiometric encountering of the allyl and thiol groups in the thin film. Therefore, we decreased the amount of PETMP to half (PETMP and AAAc in $1: 8$ ratio) to provide more alkene double bonds per -SH group so as to enhance the probability of the reaction at the thiol group sites. Interestingly, the irradiated film became dry to yield a smooth photo-cured film. The FT-IR corresponding to this composition revealed the complete disappearance of the $-\mathrm{SH}$ stretching peak, strongly corroborating the occurrence of thiol-ene click reaction (Fig. 2b).

To enable the thiol-ene chemistry for oxide nanoimprinting, a photo-curable metal-containing resin formulation was prepared by mixing titanium-allyl acetoacetate complex [Ti(AAAc) $\left.)_{4}\right]$ with PETMP and HMP. Table 1 shows the three resin formulations that were used for photo-curability studies. The first composition (TA), which does not contain PETMP could undergo [2+2] photo-addition of alkene double bonds to yield cyclobutane derivative. This composition yielded a dry film after UV irradiation. However, the film was found to be brittle and easily flaked off of the silicon wafer. This could be due to the excessive photo-curing of the four-arm alkene-functionalized $\mathrm{Ti}(\mathrm{AAAc})_{4}$ that caused residual stress in the thin film. ${ }^{53}$ In the second composition (TAP-0.5), 1/8 equivalent of PETMP with respect to $\mathrm{Ti}(\mathrm{AAAc})_{4}$ was introduced to keep the effective available thiol groups per alkene as 0.5. This formulation after UV irradiation was found to yield a dry and smooth film that did not suffer any peeling off behavior. This could be attributed to the cushioning effect provided by the optimum cross-linking of PETMP that circumvented cracking of the film.

In the third composition (TAP-1), $1 / 4$ equivalent of PETMP with respect to $\mathrm{Ti}(\mathrm{AAAc})_{4}$ was used in order to keep the thiol to alkene ratio $1: 1$. Interestingly, the film obtained with this composition was found not fully dry after $20 \mathrm{~min}$ of UV irradiation. This is similar to the poor photo-curing observed with the metal-free resin containing stoichiometric ratio of allyl to thiol. Thus, for the remaining characterization and imprinting studies, we utilized the optimized composition of TAP-0.5.

Fig. 3 shows the FT-IR analyses using TAP-0. 5 to probe the thiol-ene photo-click reaction. As seen from the spectra, the characteristic ester and keto carbonyl groups are observed at 1745 and $1718 \mathrm{~cm}^{-1}$, respectively. Compared to the metal-free resin, pristine $\mathrm{Ti}(\mathrm{AAA})_{4}$ showed two additional characteristic peaks at 1610 and $1532 \mathrm{~cm}^{-1}$ that can be attributed to the bidendate chelation of the enol form of AAAc. ${ }^{54}$ In addition, the carbon-carbon double bond of allyl group appeared at $1633 \mathrm{~cm}^{-1}$. In the case of TAP-0.5, in addition to the above mentioned stretching peaks, a new peak at $2572 \mathrm{~cm}^{-1}$ corresponding to the thiol appeared. After UV light irradiation, the thiol peak became nearly invisible, while the intensity of allyl double bond peak diminished. These changes clearly indicate the occurrence of click reaction between the thiol and allyl

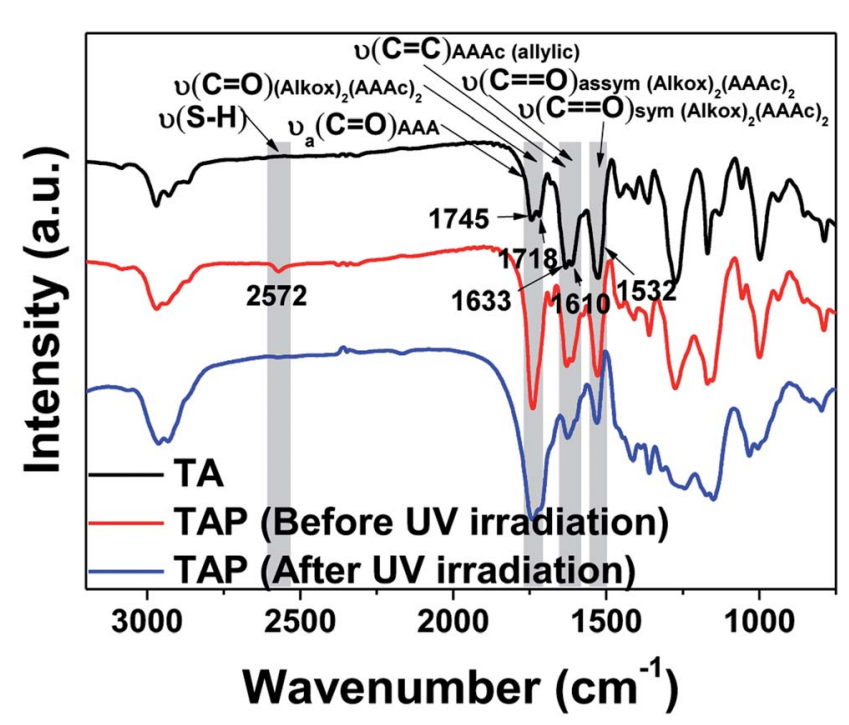

Fig. 3 FT-IR study of thiol-ene photo-addition in the titanium-containing resin TAP-0.5. 

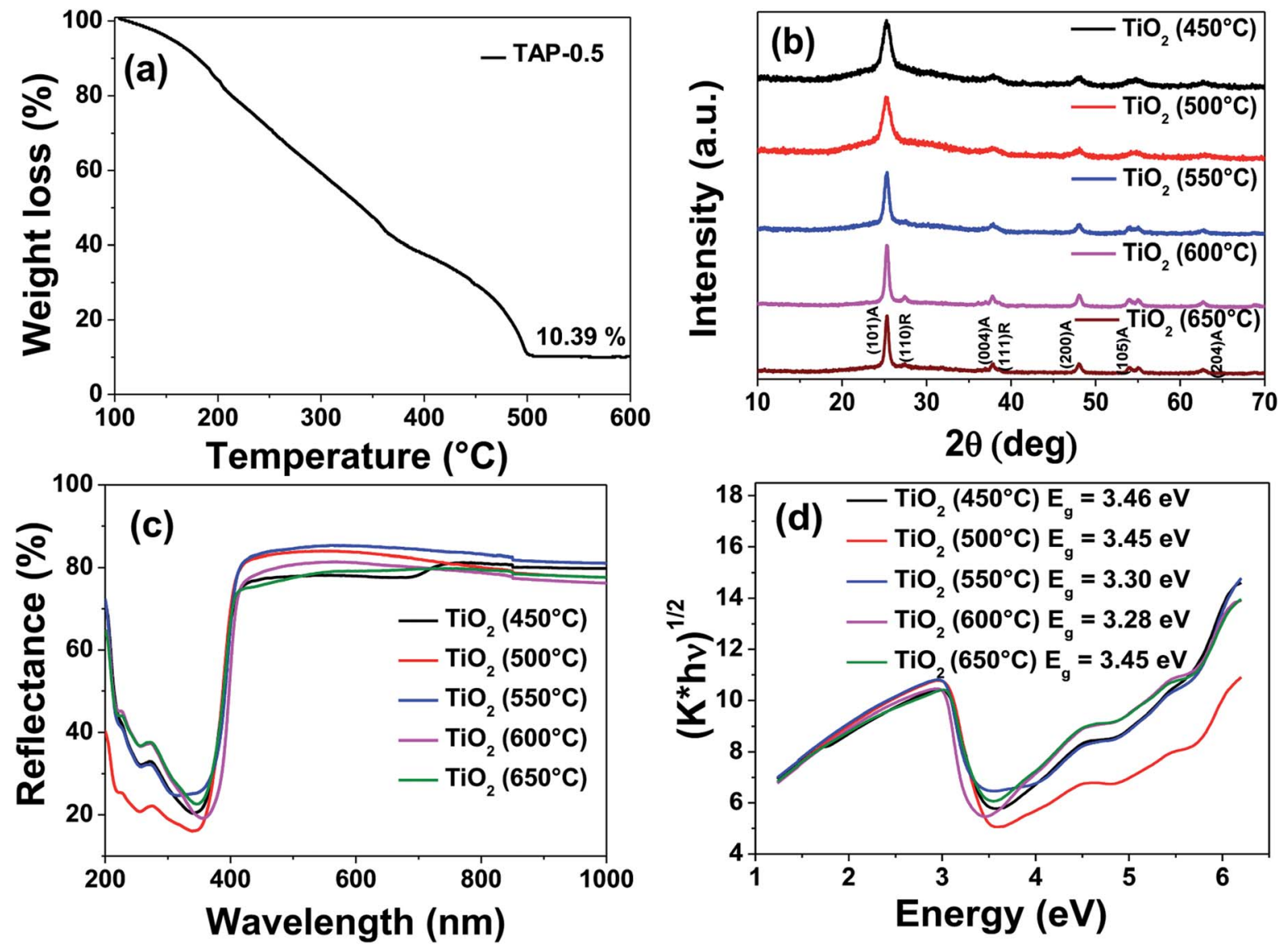

Fig. 4 (a) Thermogravimetric analysis of TAP- 0.5 resin. (b) XRD patterns of $\mathrm{TiO}_{2}$ obtained by calcination of photo-cured TAP-0.5 thin films at various temperatures. (c) Diffuse reflectance spectra and (d) the corresponding Kubelka-Munk plots of $\mathrm{TiO}_{2}$ thin films prepared at different temperatures.

groups in this system. It is noteworthy that the chelation with the metal remains intact even after the UV irradiation, which confirms that the metal is entrapped inside the cross-linked polymeric network.

Fig. 4a shows the thermogravimetric analysis (TGA) of TAP0.5 performed in air. A steady mass loss was observed between 100 and $500{ }^{\circ} \mathrm{C}$ due to the removal of residual solvent, low molecular weight monomers, and polymers. No further mass loss was observed above $500{ }^{\circ} \mathrm{C}$, suggesting the complete removal of organics present in the resin. The residual inorganic mass corresponding to $\mathrm{TiO}_{2}$ at $500{ }^{\circ} \mathrm{C}$ was found to be $10.4 \%$ which is in close agreement with the theoretical estimated value of $11.9 \%$.

To confirm the phases obtained after calcination of the UVcured TAP-0.5 films, X-ray diffraction (XRD) and Raman spectroscopy were performed. The calcination temperature was varied from 450 to $650{ }^{\circ} \mathrm{C}$ at a constant duration of $1 \mathrm{~h}$ to monitor the evolution of the phases and the corresponding XRD patterns are presented in Fig. 4b. As seen from the figure, the TAP-0.5 films heat-treated at 450 and $500{ }^{\circ} \mathrm{C}$ revealed the formation of phase pure anatase. The broad peaks indicate the nanocrystalline nature of $\mathrm{TiO}_{2}$ obtained at these temperatures. Above $500^{\circ} \mathrm{C}$, the (101) anatase peak was found to intensify with narrowing of the peak, indicating the growth of nanocrystallites to larger crystals (JCPDS 89-4921). At and above $600{ }^{\circ} \mathrm{C}$, formation of a small amount of rutile phase is indicated by the appearance of (110) plane at $2 \theta=27.4^{\circ}$. Applying the Scherrer's formula to the (101) plane of anatase $\mathrm{TiO}_{2}$, the crystallite size of the samples calcined at $450,500,550,600$ and $650{ }^{\circ} \mathrm{C}$ are calculated to be $6.7,7.1,12.0,15.7$, and $15.6 \mathrm{~nm}$, respectively. The Raman spectra of the $\mathrm{TiO}_{2}$ samples calcined at different

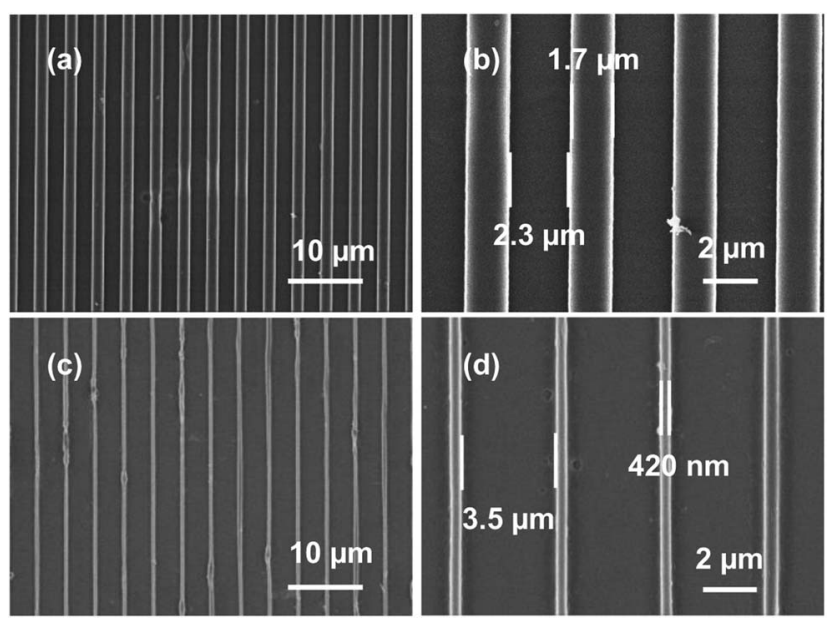

Fig. 5 FE-SEM images of imprinted lines of TAP- 0.5 ( $a$ and b) before and (c and d) after calcination. 
temperatures looked identical (Fig. S4 $\dagger$ ). A major peak at $121 \mathrm{~cm}^{-1}$ along with weak peaks at 365,482 , and $603 \mathrm{~cm}^{-1}$ were observed in all cases that can be attributed to the respective vibrational symmetries of $\mathrm{E}_{\mathrm{g}}, \mathrm{B}_{1 \mathrm{~g}}, \mathrm{~A}_{1 \mathrm{~g}}$, and $\mathrm{E}_{\mathrm{g}}$ Raman-active modes of anatase $\mathrm{TiO}_{2}{ }^{57,65}$ Due to the presence in minute quantity, the Raman peaks of rutile $\mathrm{TiO}_{2}$ were not observed in the samples calcined at 600 and $650{ }^{\circ} \mathrm{C}$.

The semiconducting nature of the oxide thin films obtained through the thiol-ene approach has been studied using diffuse reflectance spectroscopy. The percentage reflectance and their corresponding Kubelka-Munk plots of $\mathrm{TiO}_{2}$ calcined at various temperatures are shown in Fig. $4 \mathrm{c}$ and $\mathrm{d}$, respectively. The Kubelka-Munk factor $(K)$ is given by $K=(1-R)^{2} / 2 R$, where $R$ is the $\%$ reflectance. The intersection of the extrapolated linear portion of the curve in $(K \times \text { energy })^{n}$ versus energy plot with the energy axis yields the band gap of the semiconductor material. For direct band gap materials, the value of $n$ is taken as 2, while for indirect band gap material the value is taken as $1 / 2$. Since $\mathrm{TiO}_{2}$ is an indirect band gap material, the value of ' $n$ ' used was $1 / 2$. The Kubelka-Munk plots show the band gaps of $\mathrm{TiO}_{2}$ calcined at various temperatures to be in the range of 3.3 to $3.45 \mathrm{eV}$. These values agree well with the band gap of anatase $\mathrm{TiO}_{2}$ reported in the literature (3.2 to $3.4 \mathrm{eV}$ ) and thereby confirming the semiconducting nature of the obtained thin films. ${ }^{66,67}$
The metal-containing resin formulations were studied for their suitability for imprinting under laboratory conditions. A polydimethylsiloxane (PDMS) stamp was pressed over the spincoated TAP-0.5 film and irradiated with UV light. After demolding, the morphology of the photo-cured imprints before and after calcination was characterized using field-emission scanning electron microscopy (FE-SEM) and the images are shown in Fig. 5. The PDMS soft mold used in this study possessed a feature size of $\sim 2.1 \mu \mathrm{m}$ line and $\sim 1.8 \mu \mathrm{m}$ space (Fig. S5†). After patterning of TAP-0.5, the width of the imprinted line and space was found to be $\sim 1.7$ and $\sim 2.3 \mu \mathrm{m}$, respectively. The shrinkage of the imprinted lines may be attributed to the absorption of the residual solvent by PDMS mold and polymerization. Post-calcination, the lines were further shrunk to $\sim 420 \mathrm{~nm}$ and the spaces widened to $\sim 3.5 \mu \mathrm{m}$. On the whole, the $\mathrm{TiO}_{2}$ lines were shrunk to $\sim 25 \%$ compared to the as-imprinted lines, which is similar to the values previously reported PSG-based literature on $\mathrm{TiO}_{2}$ nanoimprinting. ${ }^{\mathbf{4 6}, 53-55}$ The shelf-life of the AAAc-based titanium resin was poor and thereby warrants immediate imprinting of the formulation. We speculated that this could be due to the vulnerability of titanium for hydrolysis, which was not averted by the chelated AAAc. Therefore, we briefly studied the shelf-life and imprintability of a titanium resin in which AAAc was replaced with 2(methacryloyloxy)ethyl acetoacetate (MAEAA). This formulation
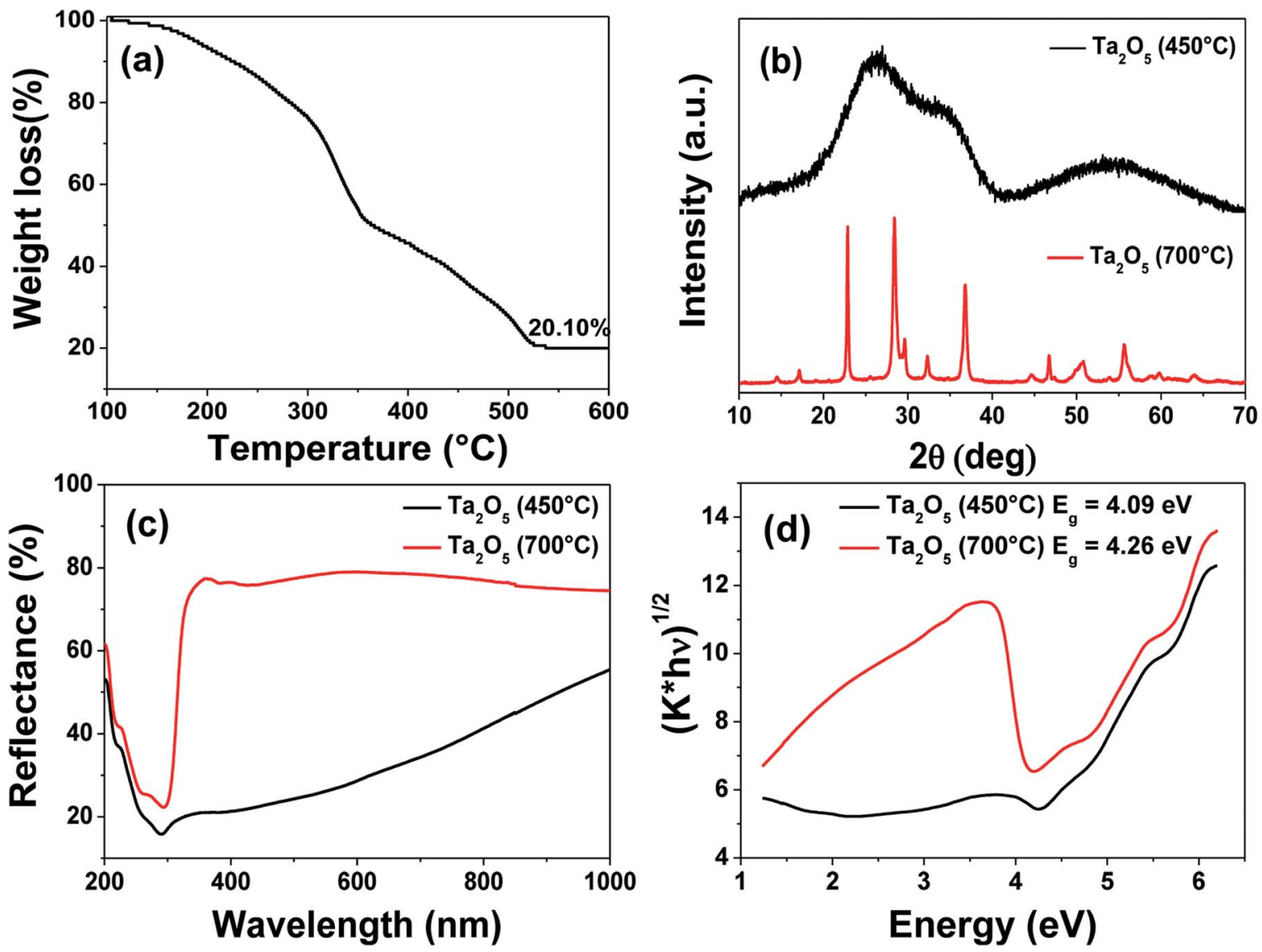

Fig. 6 (a) Thermogravimetric analysis of tantalum-containing resin. (b) XRD patterns, (c) diffuse reflectance spectra and (d) Kubelka-Munk plots of $\mathrm{Ta}_{2} \mathrm{O}_{5}$ obtained at 450 and $700{ }^{\circ} \mathrm{C}$. 
was found to have a good shelf life of more than a month. Furthermore, the successful direct imprinting using this MAEAA-based thiol-ene resin confirmed that the approach can be extended to other chelating monomers as well (Fig. S6†).

To substantiate the applicability of this thiol-ene approach for patterning other oxides, $\mathrm{Ta}_{2} \mathrm{O}_{5}$ was chosen as the candidate due to its potential applications in semiconducting devices. ${ }^{\mathbf{6 8 , 6 9}}$ Although tantalum is a pentavalent metal, we used 4 equivalents of AAAc in order to match with the corresponding titanium precursor. The resultant resin was found to have a good shelf life of more than a month. The PETMP ratio to the AAAc was fixed in the similar fashion to that used in TAP-0.5 and the FT-IR analyses before and after UV light exposure were performed (Fig. S7 $\dagger$ ). The results revealed the disappearance of the $-\mathrm{SH}$ stretching peak, indicating the occurrence of thiol-ene photoclick chemistry. Fig. 6 shows the TGA, XRD and diffuse reflectance spectra of the tantalum-containing resin. The TGA profile was observed to be in similar nature to that of TAP-0.5. The complete mass loss was found to occur at $\sim 530{ }^{\circ} \mathrm{C}$ and the residual inorganic mass corresponding to $\mathrm{Ta}_{2} \mathrm{O}_{5}$ was observed to be $20.1 \%$. The XRD pattern of $\mathrm{Ta}_{2} \mathrm{O}_{5}$ was found to be broad, indicating its amorphous nature at this temperature. Therefore, the UV-cured film was calcined at $700{ }^{\circ} \mathrm{C}$ to obtain crystalline $\mathrm{Ta}_{2} \mathrm{O}_{5}$. The XRD analysis of this sample revealed well-defined orthorhombic crystalline phase of $\mathrm{Ta}_{2} \mathrm{O}_{5}$ (JCPDS no. 79$1375) .^{70}$ The diffuse reflectance and the corresponding KubelkaMunk plots revealed a shallow spectrum for the $450{ }^{\circ} \mathrm{C}$ calcined sample, confirming the amorphous nature of the $\mathrm{Ta}_{2} \mathrm{O}_{5}$. The thin film calcined at $700{ }^{\circ} \mathrm{C}$ was found to exhibit a band gap of $4.26 \mathrm{eV}$, which is similar to that reported in the literature. ${ }^{71}$

UV imprint lithography of the tantalum-containing resin was performed and the FE-SEM images corresponding to the asimprinted and calcined samples are shown in Fig. 7. The line and space feature sizes of the as-imprinted patterns were found to be $\sim 1.7$ and $\sim 2.1 \mu \mathrm{m}$, respectively. After calcination, the lines were shrunk to $\sim 470 \mathrm{~nm}$ and the spaces extended to $\sim 3.4 \mu \mathrm{m}$. The cross-sectional view of FE-SEM images of the as-imprinted
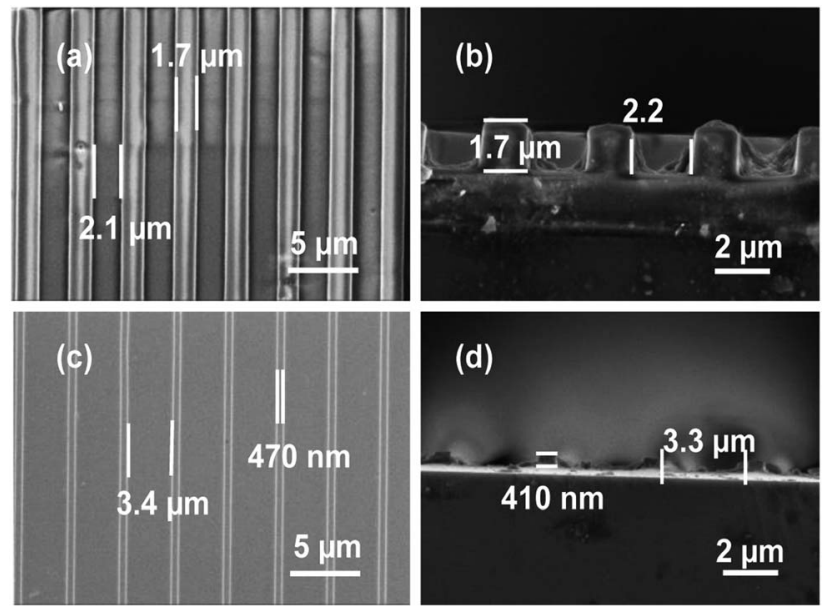

Fig. 7 Areal ( $a$ and $c$ ) and cross-sectional ( $b$ and d) FE-SEM images of as-imprinted ( $a$ and $b$ ) and post-calcined ( $c$ and $d$ ) imprints of tantalum-containing resin. and calcined samples showed the heights to be $\sim 1.7 \mu \mathrm{m}$ and $410 \mathrm{~nm}$, respectively. Thus, the lateral and vertical shrinkages were found to be $\sim 75 \%$ that is similar to the case of $\mathrm{TiO}_{2}$. The very similar imprinting behavior of tantalum-containing resin shows the capability of the thiol-ene approach to be a potential route for fabricating nanostructures of a host of oxides that could find applications in various devices.

\section{Conclusion}

In summary, thiol-ene photo-click chemistry has been successfully applied to the fabrication of metal oxide micro/ nanostructures via imprinting. When PETMP and AAAc were mixed to keep the allyl to thiol ratio as $1: 1$, the FT-IR studies revealed poor efficiency of the thiol-ene photo-click chemistry. When allyl to thiol ratio was kept as $1: 0.5$, the thiol-ene photoclick chemistry was found to be efficient under ambient conditions, possibly due to the enhanced probability of the reaction between $-\mathrm{SH}$ group and the abundant alkene. The metal-containing resin consisted of chelated titanium alkoxide with AAAc (or MAEAA) with suitable proportion of PETMP crosslinker, and HMP photo-initiator. With the optimal amount of allyl to thiol ratio as $1: 0.5$ (TAP-0.5), the resin exhibited excellent photo-curability to yield stable, crack-free, and dry thin film. The XRD and Raman analyses revealed the formation of anatase $\mathrm{TiO}_{2}$ phase at temperatures below $550{ }^{\circ} \mathrm{C}$, above which a small amount of rutile formation was confirmed with $\mathrm{XRD}$. The diffuse reflectance spectroscopy revealed the semiconducting nature of the thin film $\mathrm{TiO}_{2}$. The imprinting studies demonstrated the patternability of the titanium-containing resin to fabricate micro/nanostructures of $\mathrm{TiO}_{2}$. Successful extension of this thiol-ene photo-click chemistry for patterning $\mathrm{Ta}_{2} \mathrm{O}_{5}$ confirmed the leeway of this approach towards fabrication of various functional oxide nanostructures.

\section{Conflicts of interest}

There are no conflicts to declare.

\section{Acknowledgements}

R. G. thanks the Department of Science \& Technology (SERB/F/ 825/2014-15) for the financial aid. He also thanks Department of Science and Technology - fund for improvement of science and technology infrastructure (DST FIST; SR/FST/CSI-240/2012) to procure FT-IR, Raman microscopy, and UV-visible spectroscopy.

\section{References}

1 L. M. S. Ramirez, M. He, S. Mailloux, J. George and J. Wang, Small, 2016, 12, 3259-3269.

2 H. C. Kolb and K. B. Sharpless, Drug Discovery Today, 2003, 8, 1128-1137.

3 C. H. Kim, J. H. Bang, K. B. Hong and M. H. Park, RSC Adv., 2016, 6, 96700-96705.

4 L. Ruizendaal, S. P. Pujari, V. Gevaerts, J. M. Paulusse and H. Zuilhof, Chem.-Asian J., 2011, 6, 2776-2786. 
5 O. Z. Durham, H. R. Norton and D. A. Shipp, RSC Adv., 2015, 5, 66757-66766.

6 T. Modjinou, H. Rodriguez-Tobias, G. Morales, D. L. Versace, V. Langlois, D. Grande and E. Renard, $R S C A d v$., 2016, 6, 88135-88142.

7 Y. B. Zhou and Z. P. Zhan, Chem.-Asian J., 2018, 13, 9-19.

8 C. E. Hoyle and C. N. Bowman, Angew. Chem., Int. Ed., 2010, 49, 1540-1573.

9 A. B. Lowe, Polym. Chem., 2010, 1, 17-36.

10 A. B. Lowe, Polym. Chem., 2014, 5, 4820-4870.

11 M. J. Kade, D. J. Burke and C. J. Hawker, J. Polym. Sci., Part A: Polym. Chem., 2010, 48, 743-750.

12 J. Tan, C. Li, J. Zhou, C. Yin, B. Zhang, J. Gu and Q. Zhang, RSC Adv., 2014, 4, 13334-13339.

13 L. M. Campos, K. L. Killops, R. Sakai, J. M. Paulusse, D. Damiron, E. Drockenmuller, B. W. Messmore and C. J. Hawker, Macromolecules, 2008, 41, 7063-7070.

14 T. R. Hayes, P. A. Lyon, E. Silva-Lopez, B. Twamley and P. D. Benny, Inorg. Chem., 2013, 52, 3259-3267.

15 L. A. Connal, C. R. Kinnane, A. N. Zelikin and F. Caruso, Chem. Mater., 2009, 21, 576-578.

16 J. Xu and C. Boyer, Macromolecules, 2015, 48, 520-529.

17 A. V. Bordoni, M. V. Lombardo and A. Wolosiuk, RSC Adv., 2016, 6, 77410-77426.

18 Z. Xu, M. Yin, J. Sun, G. Ding, L. Lu, P. Chang, X. Chen and D. Li, Nanotechnology, 2016, 27, 115401-115411.

19 J. Na, Y. Kim, C. Park and E. Kim, NPG Asia Mater., 2015, 7, 217-223.

20 P. Hu, G. Du, W. Zhou, J. Cui, J. Lin, H. Liu, D. Liu, J. Wang and S. Chen, ACS Appl. Mater. Interfaces, 2010, 2, 3263-3269.

21 S. G. Kumar and L. G. Devi, J. Phys. Chem. A, 2011, 115, 13211-13241.

22 N. Tohge, R. Ueno, F. Chiba, K. Kintaka and J. Nishii, J. SolGel Sci. Technol., 2000, 19, 119-123.

23 L. Li, S. Chakrabarty, K. Spyrou, C. K. Ober and E. P. Giannelis, Chem. Mater., 2015, 27, 5027-5031.

24 M. Saifullah, K. Subramanian, E. Tapley, D.-J. Kang, M. Welland and M. Butler, Nano Lett., 2003, 3, 1587-1591.

25 D. Garoli and G. Della Giustina, Mater. Chem. Phys., 2015, 164, 63-70.

26 N. E. Voicu, M. Saifullah, K. Subramanian, M. E. Welland and U. Steiner, Soft Matter, 2007, 3, 554-557.

27 S. Lee, S. H. Jung, D. J. Kang and J. Lee, $R S C A d v$., 2016, 6, 5944-5948.

28 E. B. Duoss, M. Twardowski and J. A. Lewis, Adv. Mater., 2007, 19, 3485-3489.

29 S. Sung, S. Park, S. Cha, W. J. Lee, C. H. Kim and M. H. Yoon, RSC Adv., 2015, 5, 38125-38129.

30 L. Fu, X. Liu, Y. Zhang, V. P. Dravid and C. A. Mirkin, Nano Lett., 2003, 3, 757-760.

31 M. Su, X. Liu, S.-Y. Li, V. P. Dravid and C. A. Mirkin, J. Am. Chem. Soc., 2002, 124, 1560-1561.

32 S. H. Lim, M. Saifullah, H. Hussain, W. W. Loh and H. Y. Low, Nanotechnology, 2010, 21, 285303-285308.

33 M. Li, H. Tan, L. Chen, J. Wang and S. Y. Chou, J. Vac. Sci. Technol., B: Microelectron. Nanometer Struct.-Process., Meas., Phenom., 2003, 21, 660-663.
34 H. H. Park, D. G. Choi, X. Zhang, S. Jeon, S. J. Park, S. W. Lee, S. Kim, K. d. Kim, J. H. Choi and J. Lee, J. Mater. Chem., 2010, 20, 1921-1926.

35 S. Azimi, J. Song, C. Li, S. Mathew, M. Breese and T. Venkatesan, Nanotechnology, 2014, 25, 445301-445308.

36 M. Faustini, C. Boissiere, L. Nicole and D. Grosso, Chem. Mater., 2013, 26, 709-723.

37 E. A. Costner, M. W. Lin, W. L. Jen and C. G. Willson, Annu. Rev. Mater. Res., 2009, 39, 155-180.

38 K. J. Byeon and H. Lee, Eur. Phys. J.: Appl. Phys., 2012, 59, 10001.

39 J. G. Ok, Y. J. Shin, H. J. Park and L. J. Guo, Appl. Phys. A, 2015, 121, 343-356.

40 D. G. Choi, K. J. Lee, S. Kim, E. S. Lee, J. H. Jeong, J. Lee and J. H. Choi, RSC Adv., 2012, 2, 11035-11039.

41 D. J. Resnick, S. Sreenivasan and C. G. Willson, Mater. Today, 2005, 8, 34-42.

42 T. S. Kustandi, W. W. Loh, H. Gao and H. Y. Low, ACS Nano, 2010, 4, 2561-2568.

43 S. Takei, T. Ogawa, R. Deschner and C. G. Willson, Microelectron. Eng., 2014, 116, 44-50.

44 N. Kooy, K. Mohamed, L. T. Pin and O. S. Guan, Nanoscale Res. Lett., 2014, 9, 320-322.

45 S. H. Ahn and L. J. Guo, ACS Nano, 2009, 3, 2304-2310.

46 R. Ganesan, J. Dumond, M. S. Saifullah, S. H. Lim, H. Hussain and H. Y. Low, ACS Nano, 2012, 6, 1494-1502.

47 O. F. Gobel, M. Nedelcu and U. Steiner, Adv. Funct. Mater., 2007, 17, 1131-1136.

48 S. J. Kwon, J. H. Park and J. G. Park, J. Electroceram., 2006, 17, 455-459.

49 E. Zanchetta, V. Auzelyte, J. Brugger, A. V. Savegnago, G. Della Giustina and G. Brusatin, Microelectron. Eng., 2012, 98, 176-179.

50 J. H. Choi, H. B. Jo, H. J. Choi and H. Lee, Nanotechnology, 2013, 24, 195301-195308.

51 R. Kothari, M. R. Beaulieu, N. R. Hendricks, S. Li and J. J. Watkins, Chem. Mater., 2017, 29, 3908-3918.

52 G. Rasic and J. Schwartz, MRS Commun., 2013, 3, 207-211.

53 R. Ganesan, S. H. Lim, M. Saifullah, H. Hussain, J. X. Kwok, L. Ryan, H. A. Bo and H. Y. Low, J. Mater. Chem., 2011, 21, 4484-4492.

54 S. S. Dinachali, M. S. Saifullah, R. Ganesan, E. S. Thian and C. He, Adv. Funct. Mater., 2013, 23, 2201-2211.

55 S. S. Dinachali, J. Dumond, M. S. M. Saifullah, K. K. AnsahAntwi, R. Ganesan, E. S. Thian and C. He, ACS Appl. Mater. Interfaces, 2013, 5, 13113-13123.

56 R. Nagarjuna, S. Roy and R. Ganesan, Microporous Mesoporous Mater., 2015, 211, 1-8.

57 S. Challagulla, R. Nagarjuna, R. Ganesan and S. Roy, ACS Sustainable Chem. Eng., 2016, 4, 974-982.

58 E. C. Hagberg, M. Malkoch, Y. Ling, C. J. Hawker and K. R. Carter, Nano Lett., 2007, 7, 233-237.

59 L. M. Campos, I. Meinel, R. G. Guino, M. Schierhorn, N. Gupta, G. D. Stucky and C. J. Hawker, Adv. Mater., 2008, 20, 3728-3733.

60 H. Lin, X. Wan, X. Jiang, Q. Wang and J. Yin, Adv. Funct. Mater., 2011, 21, 2960-2967. 
$61 \mathrm{X} . \mathrm{Hu}, \mathrm{S}$. Huang, R. Gu, C. Yuan, H. Ge and Y. Chen, Macromol. Rapid Commun., 2014, 35, 1712-1718.

62 M. Pelaez, N. T. Nolan, S. C. Pillai, M. K. Seery, P. Falaras, A. G. Kontos, P. S. Dunlop, J. W. Hamilton, J. A. Byrne and K. O'shea, Appl. Catal., B, 2012, 125, 331-349.

63 L. Song, A. Abdelsamie, C. J. Schaffer, V. Korstgens, W. Wang, T. Wang, E. D. Indari, T. Froschl, N. Husing and T. Haeberle, Adv. Funct. Mater., 2016, 26, 7084-7093.

64 C. Pina-Hernandez, V. Lacatena, G. Calafiore, S. Dhuey, K. Kravtsov, A. Goltsov, D. Olynick, V. Yankov, S. Cabrini and C. Peroz, Nanotechnology, 2013, 24, 065301-065306.

65 W. Su, J. Zhang, Z. Feng, T. Chen, P. Ying and C. Li, J. Phys. Chem. C, 2008, 112, 7710-7716.
66 A. L. Linsebigler, G. Lu and J. T. Yates Jr, Chem. Rev., 1995, 95, 735-758.

67 S. Challagulla, K. Tarafder, R. Ganesan and S. Roy, Sci. Rep., 2017, 7, 8783.

68 X. Lü, S. Ding, T. Lin, X. Mou, Z. Hong and F. Huang, Dalton Trans., 2012, 41, 622-627.

69 D. Zhao, C. Zhang, H. Kim and L. J. Guo, Adv. Energy Mater., 2015, 5, 1500768.

70 S. Dhawan, T. Dhawan and A. G. Vedeshwar, J. Alloys Compd., 2016, 657, 366-371.

71 W. J. Chun, A. Ishikawa, H. Fujisawa, T. Takata, J. N. Kondo, M. Hara, M. Kawai, Y. Matsumoto and K. Domen, J. Phys. Chem. B, 2003, 107, 1798-1803. 